\title{
Stretched Lens Solar Array: The Best Choice for Harsh Orbits
}

\author{
Julie A. Rodiek ${ }^{1}$ and Henry W. Brandhorst, $\mathrm{Jr}^{2}{ }^{2}$ \\ Space Research Institute, Auburn University, AL, 36849, U.S.A. \\ and \\ Mark J. O’Neill ${ }^{3}$ \\ ENTECH, Inc., Keller, TX,76248, U.S.A.
}

\begin{abstract}
In past years, very few satellites have flown in orbits that require long durations in the Van Allen radiation belts due to the loss of power, hence lifetime, caused by the radiation. Another recurring obstacle to reliable power in space is electrostatic discharge which is most prevalent in GEO. As power levels continue to increase, arcing failures will become more prevalent. An array that can withstand high voltage, high radiation operation while being lightweight and cost efficient is necessary for the future of the space industry. The Stretched Lens Array (SLA) developed by ENTECH, Inc. is a viable candidate. This paper will discuss recent advances, past and future testing protocols, flight history, and the ability of the SLA to reliably fly in harsh orbits.
\end{abstract}

\section{Introduction}

TN past years, very few satellites have flown in orbits that require long durations in the Van Allen radiation belts 1 due to the loss of power, hence lifetime, caused by the radiation. Orbits within these radiation belts are attractive for a wide range of missions including communication, observation, and resource assessment. It is also pertinent for Solar Electric Propulsion (SEP) travel to be able to transverse through the Van Allen Belts with minimal degradation on the way to the moon or beyond.

Another recurring obstacle to reliable power in space is electrostatic discharge which is most prevalent in GEO. As power levels continue to increase, arcing failures will become more prevalent. An array that can withstand high voltage, high radiation operation while being lightweight and cost efficient is necessary for the future of the space industry. The Stretched Lens Array (SLA) developed by ENTECH, Inc. is a viable candidate. This paper will discuss recent advances, past and future testing protocols, flight history, and the ability of the SLA to reliably fly in harsh orbits.

\section{Solar Array Anomalies}

A statistical analysis of satellite anomalies performed with the use of Airclaims's Ascend SpaceTrak database ${ }^{1}$ determined that the GEO environment is especially dangerous for solar arrays. Spacecraft charging in geosynchronous orbit is a reality that can be destructive and thus negatively affect the satellite industry as a whole. Figure 1 shows that the number of satellite anomalies in GEO is significantly greater than any other orbit for the last ten years. In the last ten years only $25 \%$ of satellite launches went to GEO. However, $41 \%$ of all anomalies

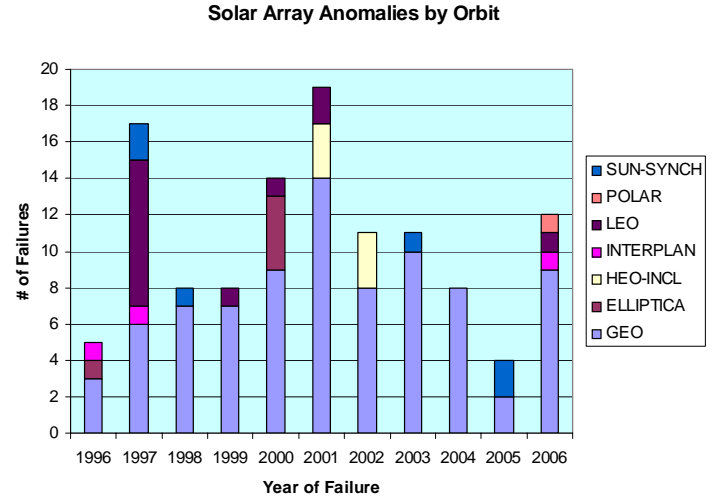

Figure 1. Satellite failures by orbit

\footnotetext{
${ }^{1}$ Director, Space Research Institute, 231 Leach Science Center, Associate Fellow.

${ }^{2}$ Research Engineer II, Space Research Institute, 231 Leach Science Center, Member.

${ }^{3}$ President, ENTECH Inc., 1077 Chisolm Trail.
} 
and failures occurred in GEO including 71\% of all solar array anomalies. The majority of these anomalies can be traced to electrostatic discharges that often occur when the satellite emerges from an eclipse period into a solar storm. Yet over the last decade, no effective solution for this problem has been implemented.

Solar array power loss by radiation degradation is another anomaly that occurs frequently. This is especially common in the high radiation environment of MEO. For SEP missions to become practical the arrays must be able to spiral out through the Van Allen Belts with minimal degradation. Additional coverglass can be added but this is usually a very costly solution due to the additional weight. The SLA can overcome these two potentially deadly failure mechanisms and still remain lightweight and cost-effective.

\section{SLA Performance Parameters}

The SLA developed by ENTECH Inc. is a space solar array that uses refractive concentrator technology to collect and convert solar energy into useful electricity. The concentrator uses a stretched Fresnel lens $(8.5 \mathrm{~cm}$ aperture width) that refracts the incident light onto high-performance multi-junction photovoltaic cells $(1.0 \mathrm{~cm}$ active width). SLA's unique, lightweight, and efficient design leads to outstanding performance ratings as shown below: ${ }^{2}$

* Areal Power Density: $>300 \mathrm{~W} / \mathrm{m}^{2}$ due to High-Performance Lenses and Cells

- Specific Power: > $300 \mathrm{~W} / \mathrm{kg}$ for a $100 \mathrm{~kW}$ Solar Array on ATK's SquareRigger Platform

* Stowed Power: $>80 \mathrm{~kW} / \mathrm{m}^{3}$ for a $100 \mathrm{~kW}$ Solar Array also with ATK’s SquareRigger Platform

- Scalable Array Power Capacity: 4 kW to 100's of kW's

* Super-Insulated Small Cell Circuit: High-Voltage (up to 600 V) Operation

* Super-Shielded Small Cell Circuit: Excellent Radiation Hardness at Low Mass

* 85\% Cell Area Savings: Up to 75\% Savings in Array \$/W Versus One-Sun Array

* Modularity \& Mass-Producibility at MW’s per Year Using Existing Processes and Capacities

The SLA's attributes match the critical requirements needed for radiation hardened satellite systems that are to transverse through the Van Allen Belts. SLA's small cell size which is $85 \%$ smaller than planar high-efficiency arrays, allows shielding and insulation to be added without detrimental mass effects. In the SLA, the entire cell and cell edges are fully encapsulated providing a sealed environment preventing the most common failure mechanism of arcing.

\section{The Truth behind Concentrator Arrays}

Concentrator arrays come with a bad name due to very expensive and grievous miscalculations in the past. However,

not all concentrators are same and therefore they should not all be disregarded. The highly publicized concentrator failures dealt with reflective photovoltaic systems. One main problem with reflector systems is shape error tolerance. Refractive concentrators minimize the effects of shape errors and provide more than 100X larger slope error tolerance than reflective or flat concentrators. ${ }^{3}$ This can be seen in figures 2 and 3.

Another shortcoming of reflector concentrator systems is non-uniform irradiance. This means that the cell with the least illumination will limit the current of the cells that are connected in series with it. This can have a significant dampening effect on the power output of the solar array. The SLA avoids these problems entirely by having the same focal line on every cell in every series string thus making the

Symmetrical-Refraction Lens Provides Maximum Performance and Shape Error Tolerance

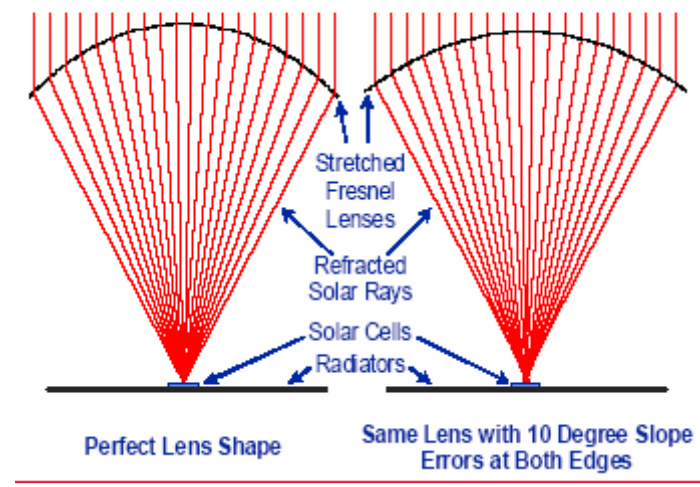

Figure 2. Shape error tolerance for a refraction system.



Figure 3. Distortion of a reflection system 
current output essentially identical in every string.

The thermal challenge of removing heat from the PV panel is another shortcoming of the reflector system. The radiator area needs to be the same size as the aperture area of the optics. This can be quite costly due the increased weight. High temperatures can cause outgassing leading to damage to the array and severe power degradation as was seen in previous attempts. Due to the small aperture width of $8.5 \mathrm{~cm}$, the SLA is able to get by with a small radiator thickness. This limits both the amount of waste heat and the distance it must be conducted. The SCARLET array, a predecessor of SLA, remained at the predicted temperature throughout its mission and had no outgassing.

A query of the Airclaims's Ascend SpaceTrak database on concentrator failures showed the only related fault affected the fleet of Boeing 702/GEM satellites. Degradation in power occurred at a faster rate than expected causing satellite life expectancy to be downgraded from 15 years to 6.75 years. This was believed to be caused by a 'fogging' effect caused by outgassing. ${ }^{1}$ This is due to the heating effect that these reflective concentrators have on the solar arrays. The geometry of these concentrators created a heat trough which caused higher temperatures and outgassing of sealants leading to a 'fogging' affect that lowered power production. This is NOT seen in refractive systems such as the SLA. The SLA concentrator system provides reliable power and should not be mistaken for its predecessors.
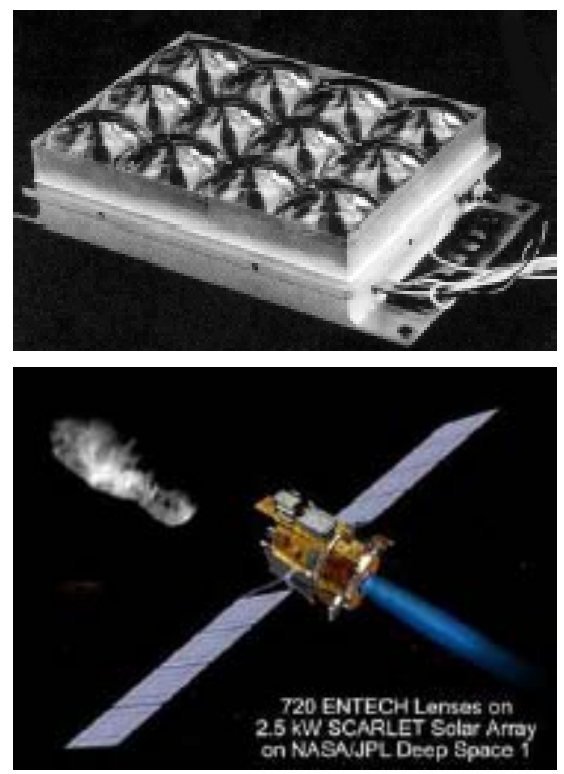

Figure 4. Top photo is the PASP+ module, bottom photo is the SCARLET Deep Space 1 array

\section{SLA Flight Background}

A mini-dome lens concentrator flown on the PASP-Plus mission in 1994 was the first refractive concentrator array. It provided the best performance and least degradation of 12 advanced solar array experiments that flew on the mission in a high radiation orbit. ${ }^{4}$ SCARLET, a line focused concentrator, evolved from this and was launched in 1998 on Deep Space 1 and performed flawlessly on a 38 month mission ${ }^{5}$. Both PASP+ and SCARLET are pictured in Fig. 4. The stretched lens array is based on SCARLET and retains the essential power-generating elements but eliminates the complexity, fragility, expense, and mass of the glass arches by incorporating pop-up arches ${ }^{6}$. Flexible blanket and rigid panel versions of the SLA have been developed and tested over the last decade. A $3.75 \mathrm{~kW}$ scale $(2.5 \times 5.0 \mathrm{~m})$ building block of the Stretched Lens Array on the SquareRigger platform has been successfully demonstrated as seen in Fig. 5. This module is sized to produce $3.75 \mathrm{~kW}$ and weighs only about $10 \mathrm{~kg}$ demonstrating the specific power goal of $>300 \mathrm{~W} / \mathrm{kg}$ is achievable.

\section{Radiation Degradation}

Radiation degradation affects all satellites that fly in orbits that require long durations in the Van Allen radiation belts. Additional coverglass can be added to arrays to protect against radiation damage but this is usually a very costly solution due to the additional weight. In the case of the SLA, radiation shielding can be increased with little impact on the SLA's mass, hence providing a "super shielded" system. This is due to its $8 \mathrm{X}$ concentration which reduces the area, hence mass, of solar cells needed for the desired power range.

Theoretical modeling and ground testing has been completed to test the survivability of the SLA and planar arrays in high radiation environments. To understand and compare the various radiation environments for these orbits, simulations have been run using The European Space Environment Information System (SPENVIS). The SPENVIS model provides the 1 $\mathrm{MeV}$ equivalent electron radiation doses for given orbits and durations. This information, in conjunction with a standardized chart of power degradation of solar cells with electron fluence, permits calculation of the power degradation of the solar cell as a function of cover glass thickness.

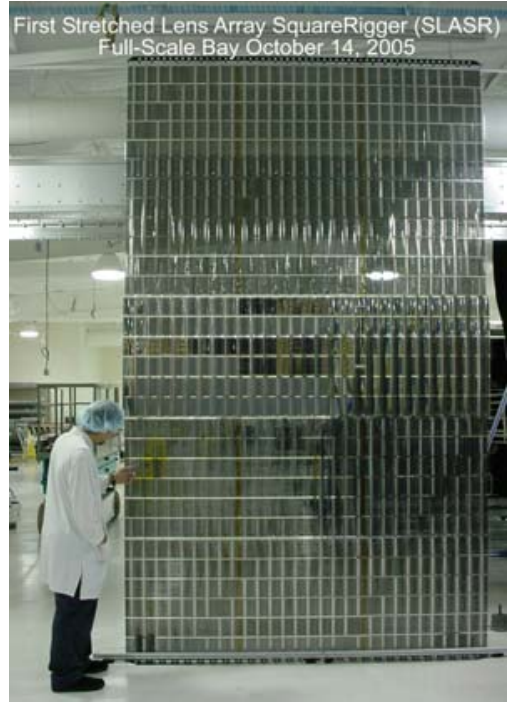

Figure 5. Full scale SLASR panel 
A high radiation orbit of $5000 \mathrm{~km}$ with a 28 degree inclination angle was chosen as an example. Next the mass of the cover glass material must be considered to allow calculation of the end-of-life (EOL) specific power for the array. The peak EOL specific power values for each time period have been obtained for both the SLA and a planar array as shown in Fig. 6. This assumes a beginning of life areal power density of $300 \mathrm{~W} / \mathrm{m}^{2}$ which is comparable to today's SLA. Note that SLA offers more than a 3X advantage over the planar array for 1 year on the time scale, and a $4 \mathrm{X}$ advantage over planar for 10 years on the time scale, for this example case $(5,000 \mathrm{~km}$ altitude, 28 degree inclination, circular orbit). SLA's advantage over planar is apparent especially in high radiation missions. Figure 7 shows the SLA advantage over a planar array by displaying the areal power density variation for the heaviest SLA analyzed versus the lightest one-sun array analyzed. It is important to note that the heaviest SLA is $14 \%$ lighter than the lightest one-sun array, thus the remaining power advantage of SLA is spectacular. SLA's advantage over planar will grow even larger for higher radiation missions.

The solar array degradation for a LEO to GEO tug mission and a lunar cargo tug has also been calculated by SPENVIS simulations as seen in Fig. 8. The SLA uses more protective cover glass to reduce the radiation damage yet incurs only a small mass penalty. Comparisons of the end-oflife specific power between a SLA and a planar array are presented for an orbital transfer mission from LEO to GEO in Fig. 9. A 100 kWe SLA, adequately shielded with a 20 mil coverglass, will still have a specific power of $260 \mathrm{~W} / \mathrm{kg}$ after seven round-trip LEO-GEO missions. A conventional planar one-sun array with the same amount of shielding would only have $70 \mathrm{~W} / \mathrm{kg}$ after such a mission. This incorporates satellites being transferred in both directions with a tug mass of $1000 \mathrm{~kg}$. SLA's advantage over planar arrays in specific power is shown once again.

Ground testing must also be performed to see how the array's components stand up to radiation exposure. Combined electron and proton testing has been conducted at NASA Marshall Space Flight Center. Testing has shown that the silicone lens material of the SLA can tolerate $5 \times 10^{10}$ rads of combined electron and proton exposure with only minor degradation. This is equivalent to 10 years on GEO using the current AE8/AP8 environments. Spectral transmittance data from NASA MSFC testing of lens material with UV-rejection coatings shows no damage after more than 1000 equivalent sun hours of combined vacuum ultraviolet (VUV) and near ultraviolet (NUV) exposure. Space lens material tests were performed on the MISSE 1 and MISSE 5 flight experiments that spent 48

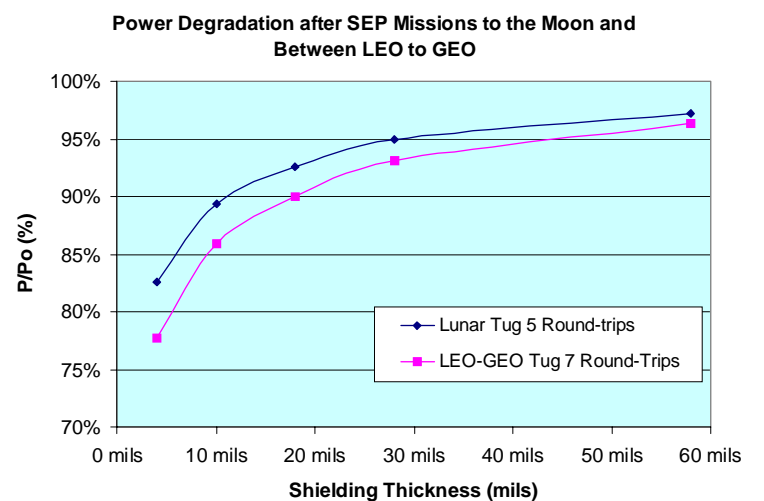

Figure 8. Power degradation of two SEP tug missions

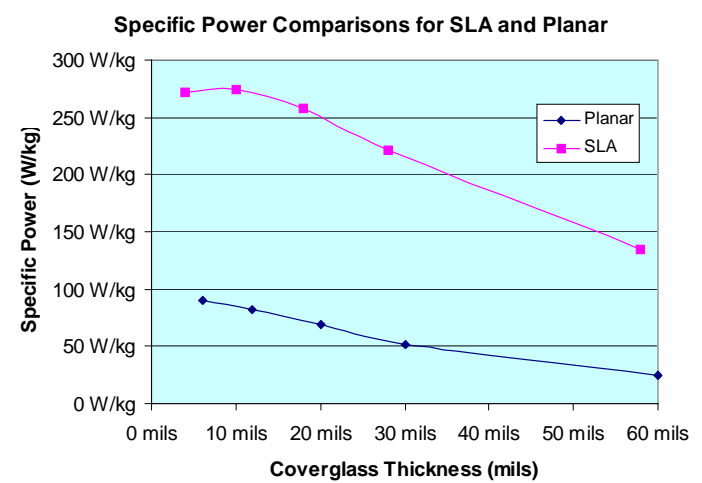

Figure 9. Specific power comparison for SLA and Planar Arrays 
months and 12 months, respectively, on the ISS exposed to sunlight. There is no available data yet for the MISSE 5 experiments, but for MISSE 1 the UVR-coated silicone lens material held up very well with very little degradation. All aspects of the SLA have tested durable to the space environment.

\section{High Voltage Operation}

Another recurring obstacle to reliable power in space is electrostatic discharge seen most commonly in high voltage operations such as high power systems or SEP. The SLA can be specifically optimized for SEP by the ability to direct-drive Hall-effect thrusters. This technology designed by NASA Glenn can minimize the inefficiency, mass, cost and complexity of the power management and distribution interface between the solar array and electric thruster. ${ }^{9}$ The initial drawback is that the solar array must be able to operate at the voltage level needed to drive the electric thruster. This voltage is much higher than the present operation voltage of space solar arrays of $100 \mathrm{~V}$. Serious discharge, arcing, and ground-fault problems have occurred on orbit with even the present operating voltage. SLA overcomes this challenge by fully encapsulating the entire cell circuit to create a sealed environment. This can be accomplished without a huge mass penalty due to the $8 \mathrm{X}$ concentration and fewer cells needed to provide the same amount of power. The SLA's inherent design also protects against electrostatic discharge. The lens is several inches away from the cells and substrate and arcing cannot occur across that distance. This solves the problems associated with corona discharge in long term missions. Other array designs do not guard against this potential failure mechanism.

The SLA module has been tested to voltages over $1000 \mathrm{~V}$ while under hypervelocity particle impact in a plasma environment with no arcing. Micrometeoroid impacts on solar arrays can lead to arcing if the spacecraft is at an elevated potential. Therefore, hypervelocity testing of the solar array is necessary. A concentrator solar cell module supplied by ENTECH, Inc was tested at Auburn University's Hypervelocity Impact Facility. The module consisted of a string of concentrator multijunction solar cells in series completely covered with cover glass. The overhang extended well beyond the cell boundaries and was also filled with silicone providing a sealed environment. The test sample in the last test is shown in Fig. 10. No surface arcs occurred over the sample despite visible particle impact penetrations of the covers. Additional tests were performed with the stretched lens in place over the samples, and the lens provided excellent shielding of the cell circuits. The sample was also exposed to rear-side impact test shot with bias voltage at $-1027 \mathrm{~V}$. Although there were many impacts no arcing was observed.

Furthermore, array segments are under test for corona breakdown that can become a critical issue for long term, high voltage missions. The SLA design is suited for high voltage operation because the entire cell and cell edges are fully encapsulated by a cover glass that overhangs the perimeter and the silicone adhesive covers the cell edges, Thus it provides a sealed environment which limits the possibility of electrostatic discharge. ENTECH has fabricated and tested a number of such single-cell SLA receiver samples at very high voltage levels $(2,250$ to $4,500 \mathrm{~V})$ in an underwater hi-pot test for very long periods of time. Auburn University has conducted similar tests in vacuum using the same type of fully encapsulated receiver samples. These tests are being conducted using the guidelines found in ESA's IEC International Standard \#343 (1991): “Recommended test methods for determining the relative resistance of insulating materials to breakdown by surface discharges." 10 The samples underwent testing at 2,250 V for ten and a half months and showed no change. Due to the SLA's inherent protection against electrostatic discharge it is especially well suited for electric propulsion missions. The SLA is also fully compliant with the new NASA-STD-4005 Low Earth Orbit Spacecraft Charging Design Standard.



\section{SEP Costs Analysis}

SEP travel holds great potential in missions to the moon, Mars, or beyond. Other uses for SEP include a LEO-toGEO orbit-raising mission for a GEO communication satellite, or an orbit-changing maneuver for a military satellite, or even returning a problematic satellite back to earth. Economic analyses of SLA-powered SEP tugs have been conducted by a number of organizations, including NASA, Orbital Sciences, Aerojet, and Ad Astra. These independent analyses show that SLA-power SEP tugs can provide billions of dollars in savings compared to conventional chemical propulsion systems in transporting cargo from the Earth to the Moon, or from the Earth on to 
Mars. One study showed SLA with SEP could save NASA > \$10 billion for lunar exploration cargo transportation.,8 A SLA-powered SEP approach for delivering 110 metric tons of cargo to the lunar surface over a five-year period will save about 350 metric tons of launch mass compared to a conventional chemical approach, comprising \$3.5 Billion in launch cost savings alone. These potential multi-billion-dollar savings could be the key to minimizing the costs of NASA's bold exploration goals to the Moon, Mars, and the solar system.

\section{Future Flight Experiment}

Under an MDA-sponsored Phase II STTR program, ENTECH and Auburn University have fabricated and delivered a near term flight experiment for integration into the main solar array for the Naval Research Lab’s TacSat IV spacecraft, which is scheduled to launch next year. This flight experiment is known as the Stretched Lens Array Technology Experiment for TacSat IV (SLATE-T4). SLATE-T4 is shown in Fig. 11. TacSat IV is intended to fly in a high radiation orbit, providing valuable solar cell degradation information along with proving flight validation for the Stretched Lens Array. The SLA receiver includes a 0.5 mm-thick ceria-doped cover glass (made by QioptiQ) and the lens incorporates ENTECH's proprietary thick parquet coating, applied by Ion Beam Optics. These will provide protection from degradation due to the effects of solar ultraviolet radiation (UV), atomic oxygen (AO), and low-energy charged particles.

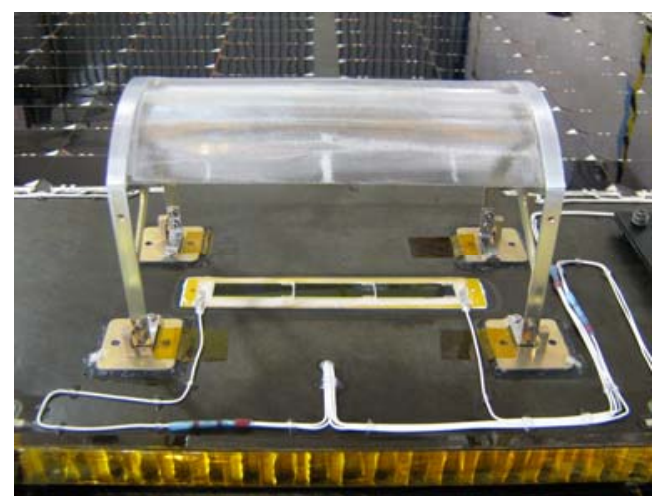

Figure 11. SLATE-T4 Parquet-Coated Lens and Three-Cell Photovoltaic Receiver Mounted on TacSat IV.

\section{$X$. Future SLA Testing}

Fig. 12 shows a schematic of a typical SLA-SEP mission with the spacecraft in earth orbit. ${ }^{3}$ The array will point toward the sun while the spacecraft orbits the earth, and some interaction will take place between the array and the thruster plume, especially at the inner corners of the array as these move through the outer regions of the plume. Thus, in addition to providing high efficiency, low mass, and radiation-hardness, the SLA must also tolerate plume interactions with the thruster.

Future SLA testing will consist of a direct drive experiment of a high-voltage (600 V open-circuit) SunLine concentrator photovoltaic array using multijunction-cell technology with a Russian T-100 Hall thruster. This is the next step under a Phase II STTR with NASA Glenn for the development of SLA hardware for SEP missions and will be performed at Auburn

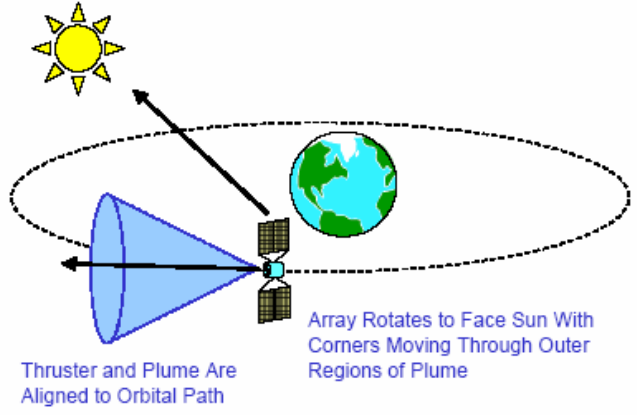

Figure 12. Typical Solar Electric Propulsion Mission Schematic. ${ }^{3}$
University. The goal of this demonstration is to prove reliable operation of a Hall thruster from a high voltage concentrator array. Testing will include the addition of Stretched Lens Array hardware in the chamber at Auburn University to measure plume impingement effects at various positions relative to the exhaust axis of the thruster. A schematic of planned testing can be seen in Fig. 13.

The high-voltage array shown in Fig. 14 was transported from ENTECH to Auburn where it is being interfaced with the Hall-effect thruster in the large vacuum chamber shown in Fig 15. The array uses two of ENTECH's color-mixing lenses to focus sunlight onto two photovoltaic receivers each using 240 series-connected triple-junction Spectrolab cells to provide $600 \mathrm{~V}$ output at open-circuit conditions. The peak power point is around $500 \mathrm{~V}$, and the total power output of the array is over $1 \mathrm{~kW}$



Figure 13. Schematic of planned direct-driven HET and SLA test configuration. 
under clear sky conditions. ${ }^{3}$ The Russian thruster shown in Fig. 15, is a Model T-100 SPT, designed and constructed by the Keldish Research Center (KeRC), and capable of operating up to $1.3 \mathrm{~kW} .^{3}$ This thruster is on loan to Auburn from NASA Glenn. Testing will start in the next few months.

\section{Conclusion}

The SLA is an optimal array for missions in harsh orbits because of its unique portfolio of attributes. Ground testing has shown it is durable and can withstand high voltage and high radiation environments. Radiation modeling has been completed with SPENVIS to show SLA's $3-4 \mathrm{x}$ advantage over planar arrays in specific power and areal power density. Future testing efforts and flight experiments have been discussed that will continue to prove the benefits of the SLA. It is ideal for SEP travel to the moon and beyond or for satellites located in the Van Allen Belts. It is the array of the future.

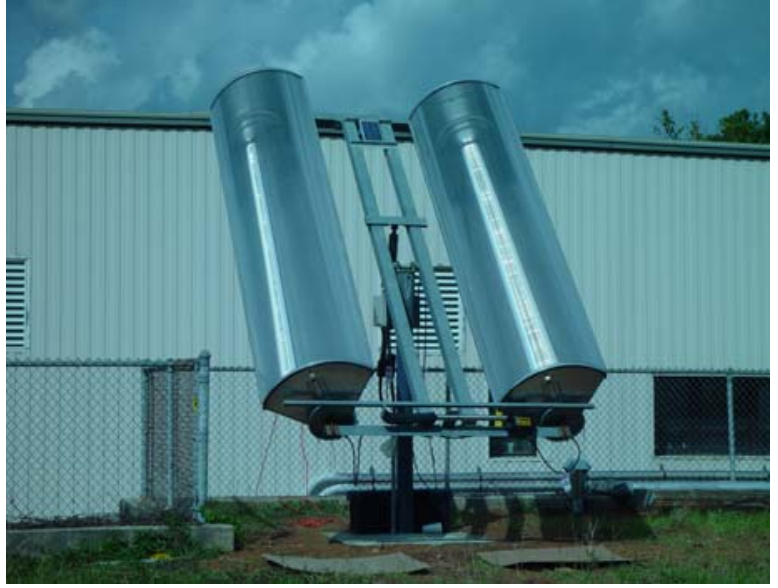

Figure 14. SunLine Concentrator array

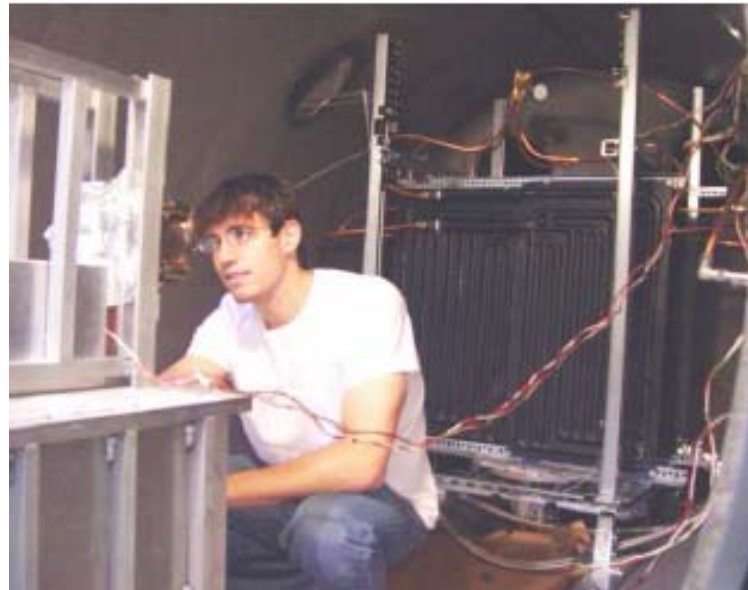

Figure 15. Auburn student inspects Russian T100 thruster

\section{Acknowledgments}

The authors gratefully acknowledge the technical and financial support from all of our partners and collaborators on SLA development, including NASA, MDA, NRL, ATK, EMCORE, Ion Beam Optics, Thin Film Technologies, QioptiQ, Aerojet, Ad Astra, Orbital Sciences, Boeing, Rocketdyne, et al., and our staffs at ENTECH and Auburn.

\section{References}

${ }^{1}$ Airclaims Ascend SpaceTrak Database, www.ascendspacetrak.com/Home

2 O’Neill, M.J., Piszczor, M., Brandhorst, H., McDermott, P., and Lewis, H., "Stretched Lens Array (SLA) for Solar Electric Propulsion (SEP), $20^{\text {th }}$ Space Photovoltaic Research and Technology Conference, Cleveland, Ohio, 2007.

${ }^{3}$ O'Neill, M.J., "Silicon Low-Concentration, Line-Focus, Terrestrial Modules," Chapter 10 in Solar Cells and Their Applications, John Wiley \& Sons, New York, 1995.

${ }^{4}$ Curtis, H. and Marvin, D., "Final Results from the PASP Plus Flight Experiment," 25th IEEE PVSC, Washington, 1996.

${ }^{5}$ Jones, P.A., Allen, D.M., Murphy, D.M., Piszczor, M.F., “The SCARLET Light Concentrating Solar Array,” 25th IEEE PVSC, Washington, 1996.

${ }^{6}$ O’Neill, M.J., “Color-Mixing Lens for Solar Concentrator System and Methods of Manufacture and Operation Thereof,” U.S. Patent 6,031,179, 2000.

${ }^{7}$ Kosmann, W., “A Solar Electric Propulsion Lunar Exploration Architecture Evaluation,” 57th International Astronautical Congress (IAC), Valencia, Spain, 2006.

${ }^{8}$ M.J. O’Neill and M.F. Piszczor, “Stretched Lens Array (SLA) Solar Electric Propulsion (SEP) Space Tug: SLA-SEP Offers Multi-Billion-Dollar Savings Delivering Lunar Exploration Cargo,” 4th World Conference on Photovoltaic Energy Conversion (WCPEC), Hawaii, 2006.

${ }^{9}$ Hamley, J. A., Sankovic, J. M., Lynn, P., O’Neill, M. J., Oleson, S. R., “Hall Thruster Direct Drive Demonstration,” 33rd AIAA/ASME/SAE/ASEE Joint Propulsion Conference, Seattle, 1997.

${ }^{10}$ IEC International Standard \#343 (1991): "Recommended test methods for determining the relative resistance of insulating materials to breakdown by surface discharges.” 